\title{
Antecedentes preliminares sobre cultivo vegetativo de Ahnfeltia plicata (Hudson) Fries, 1836 en canales subantárticos magallánicos del sur de Chile
}

\author{
Records preliminaries on vegetative cultivation of Ahnfeltia plicata (Hudson) \\ Fries, 1836 in sub-antarctic magellanic channels of southern Chile
}

Jaime Ojeda ${ }^{1,2}$, Sebastián Rosenfeld ${ }^{1,2}$, Nicolás Vega ${ }^{1} \&$ Andrés Mansilla $^{1,2}$

\section{Resumen}

La agarofita Ahnfeltia plicata es considerada una macroalga con alto potencial comercial en el mercado de ficocoloides, porque posee una agarosa neutra sin grupos sulfatos, esto abre la necesidad de mayor información sobre su cultivo. No obstante, aspectos como crecimiento en biomasa aún no han sido evaluados en Chile, siendo necesarios para un potencial cultivo. El objetivo de este trabajo fue evaluar el crecimiento vegetativo con experimentos a pequeña escala en hatchery mediante la incorporación de fertilizantes comerciales y una evaluación del crecimiento con un sistema de cultivo en mar (seno Skyring). Los resultados de cultivo de $A$. plicata en hatchery muestran que la mejor tasa de crecimiento diario en biomasa fue de 0,04 \% día para el tratamiento con Provasoli. El crecimiento vegetativo de cultivo en mar inicio con 1,2 g/ individuo y finalizó con una biomasa promedio de 2,2 g/individuo, tras 9 meses de cultivo Long-line. Esto representó un crecimiento total por talo de 0,9 g en 9 meses. La mayor tasa de crecimiento diario se observó en diciembre $(0,52 \%)$. La sobrevivencia fue de un $100 \%$. Al séptimo mes de cultivo el $56 \%$ de los talos presentaron estructuras reproductivas (nematecios). Esta investigación es la primera experiencia cultivo a pequeña escala en hatchery y mar en Chile de A. plicata. La evaluación de su crecimiento muestra que A. plicata presenta un lento aumento de biomasa en el tiempo, pero con baja mortalidad. Es fundamental continuar realizando nuevas estrategias de cultivo que permitan el uso sustentable de esta agarofita en el sur austral de Chile.

\section{Palabras claves:}

Agarosa, crecimiento vegetativo, cultivo de macroalgas, subantártico.

\section{Abstract}

The agarophyte Ahnfeltia plicata is considered a high commercial potential macroalgae market phycocolloids, because it has a neutral agarose without sulfate groups, this opens the need for more information on its cultivation. However, aspects about biomass growth have not been evaluated in Chile, being necessary for a potential culture. The aim of this study was to evaluate the vegetative growth in hatchery by using commercial fertilizers and an evaluation of growth with a sea culture system (Skyring). The results in hatchery showed that the best daily biomass growth rate was $0,04 \%$ for treatment with Provasoli. In the sea culture system the individuals starts with an average of 1,2 $\mathrm{g}$ /individual and finished with an average biomass of $2,2 \mathrm{~g} /$ individual, after 9 months of cultivation in the sea. This represents a total growth of $0.9 \mathrm{~g}$ per tally in 9 months. The

\footnotetext{
1 Laboratorio de Macroalgas Antárticas y Subantárticas, Casilla 113-D, Departamento de Ciencias, Universidad de Magallanes. $\gg$ jaimeojedavillarroel@yahoo.es, rosenfeld. sebastian@yahoo.com, niflevo@gmail.com, andres. mansilla@umag.cl

2 Instituto de Ecología \& Biodiversidad (IEB), Casilla 653, Santiago, Chile.
} 
highest growth rate was observed in December $(0,52 \%)$. Survival was $100 \%$. In the seventh month of culture $56 \%$ of the thalli showed reproductive structures (nematecios). This research is the first small-scale cultivation experience of $A$. plicata in hatchery and sea in Chile. The evaluation of his growth shows that $A$. plicata has a slow increase in biomass over time, but with low mortality. It is essential to continue making new farming strategies that allow sustainable use of this agarophyte in the Southern part of Chile.

\section{Key words:}

Agarose, Vegetative Growth, Cultivation of Macroalgae, Sub-Antarctic.

\section{INTRODUCCIÓN}

Las algas marinas presentan una gran importancia ecológica y socio-ecológica, facilitando refugio, hábitat y sitios de reproducción para diversos organismos vegetales y animales tanto invertebrados como vertebrados (e.g., Camus \& Barahona, 2002; Hori et al. 2006); también son utilizadas como fuente de alimento directo para el consumo humano (Astorga-España \& Mansilla, 2013) o para la extracción de polisacaridos como carragenanos, alginatos, fucoidanos y agarosa (Mansilla et al. 2012; Morya et al. 2012). En el caso de Chile, las principales algas de importancia económica han sido de la división Rhodophyta, de las cuales las más cosechadas han sido Sarcothalia crispata, Mazzaella laminarioides, Gigartina skottsbergii, Gelidium lingulatum, Chondracanthus chamissoi $y$ Gracilaria chilensis (Buschmann et al. 2001). Para estas dos últimas macroalgas, el desarrollo del cultivo mediante propagación vegetativa ha potenciado eficientemente su producción de biomasa. Por ejemplo, en $C$. chamissoi se han realizado experiencias de crecimiento de tejidos desde frondas reproductivas y no reproductivas (Bulboa \& Macchiavello, 2006). Mientras que la agarofita $G$. chilensis es el caso más emblemático en Chile, donde el cultivo artificial mediante propagación vegetativa ha sido desarrollado con técnicas de siembra, cosecha y metodologías de poda (e.g., Westermeier et al. 1991). Otra agarofita cosechada en menor medida en Chile es Ahnfeltia plicata (Buschmann et al. 2001; Mansilla et al. 2012), la cual en la actualidad no presenta una pesquería efectiva en la región de canales y fiordos Magallánicos.

A. plicata es considerada una de las agarofitas con más potencial comercial en el mercado de ficocoloides a nivel mundial (Maggs \& Puechel, 1989), esto por su alta calidad de agarosa y sin grupos sulfatos (Matsuhiro et al. 2014). La distribución de las poblaciones es circumpolar en el hemisferio Norte y Sur (Mansilla et al. 2013). En Chile su distribución se encuentran principalmente referida para la zona de canales y fiordos Magallánicos, donde las poblaciones más conspicuas han sido observadas en la zona del seno Skyring (Mansilla et al. 2013). Estas poblaciones crecen sobre sustrato rocoso en zonas con alto hidrodinamismo, donde la morfología de los gametofitos está compuesta por filamentos erectos que crecen desde una fase crustosa asociada al sustrato rocoso (Mansilla et al. 2013). El principal conocimiento ecológico y bioquímico de las poblaciones de A. plicata en seno Skyring radica principalmente en dos puntos: i) Son poblaciones perennes, donde la mayor biomasa se encuentra durante otoño con un promedio de 281,64 g $\pm 23,21 \mathrm{~g}$ de biomasa húmeda en $625 \mathrm{~cm}^{2}$, también durante esta estación aumenta la biomasa de individuos no reproductivos (Mansilla et al. 2013). ii) El análisis bioquímico de la agarosa, muestra que posee propiedades anticoagulantes y antioxidantes, la cual está desprovista de sulfatación y su agarosa es neutra (Matsuhiro et al. 2014); Este polisacárido constituye un material muy relevante para la preparación de derivados solubles y sus aplicaciones tienen un potencial biotecnológico (Matsuhiro et al. 2014). No obstante, uno de los mayores problemas que presenta A. plicata para posicionarse como una agarofita con gran potencial en el mercado de ficocoloides, es su bajo desarrollo del cultivo debido a su lento crecimiento. Por ejemplo, Chen (1977) trabajo con su crecimiento en el hemisferio norte $y$ observó que al cabo de un periodo de tres años de cultivo en laboratorio, logro obtener esporas de A. plicata producidas desde tetraesporofitos, los cuales posteriormente germinaron formando talos crustosos, y que finalmente desarrollaron 
talos erectos. Por otro lado, en poblaciones del hemisferio Sur, se han realizado esfuerzos de cultivo en laboratorio utilizando distintos tipos de fotoperiodo, uso de fertilizantes $y$ reguladores de crecimiento como hormonas para micropropagación (ver Villanueva et al. 2013; Mansilla et al. 2013), estos experimentos han demostrado que existe un efecto positivo de las hormonas sobre el crecimiento de talos de $A$. plicata, pero no la suficiente para desarrollar técnicas de micropropagación masiva (ver Mansilla et al. 2013).

En este contexto, las experiencias de cultivo de A. plicata se han desarrollado utilizando técnicas de cultivo en laboratorio, pero aún no existen experiencias que muestren su crecimiento vegetativo en condiciones de cultivo en hatchery y en el mar, el cual es requerimiento necesario para determinar si A. plicata presenta un potencial de cultivo, que permita disponer de biomasa, a través de un desarrollo sustentable y no solo a partir de biomasa de sus poblaciones naturales. El objetivo de esta investigación, fue evaluar el crecimiento vegetativo de A. plicata bajo condiciones de cultivo en hatchery y en mar (Seno Skyring), lo que permitirá entregar antecedentes preliminares para un potencial cultivo de A. plicata en la región de Magallanes. Esto representa una iniciativa para fortalecer la industria de ficocoloides en Chile, ya que $A$. plicata posee una agarosa neutra desprovista de grupos sulfatos, lo que representa un potencial económico para Magallanes.

\section{MATERIALES Y MÉTODOS}

\section{Sitio de colecta}

Individuos de A. plicata fueron colectados, mediante buceo autónomo a profundidades de 1 a 3 metros, desde una pradera natural localizada en seno Skyring (52 37'S; 71 $48^{\circ} \mathrm{W}$ ), Región de Magallanes, Chile, (Fig. 1). Estos fueron almacenados en contenedores térmicos con agua de mar para evitar estrés, y así trasladarlos hasta el Laboratorio de Macroalgas Antárticas y Subantárticas en la Universidad de Magallanes. Aquí fueron seleccionaron sólo talos vegetativos vivos (con ausencia de estructuras reproductivas) y sin tejido necrosado. A su vez, se realizó la limpieza de los talos extrayendo el sedimento y organismos asociados, como invertebrados o algas epífitas. Finalmente, estos talos fueron utilizados en las posteriores experiencias de cultivo.

\section{Cultivo en hatchery}

El experimento de cultivo en hatchery de A. plicata fue realizado en Centro de Cultivo y Acuario Bahía Laredo de la Universidad de Magallanes (ver Fig. 1). Aquí se efectuaron tres tratamientos: i) Control (talos de A. plicata cultivados en agua de mar sin incorporación de nutrientes artificiales); ii) talos de A. plicata cultivados en agua más la incorporación de medio de cultivo Provasoli; y iii) talos de $A$. plicata cultivados en agua con incorporación fertilizantes comerciales. Cada uno de estos tratamientos poseía 3 réplicas y en cada replica se incorporó $5,2 \mathrm{~g}$ de peso húmedo de A. plicata de tejido vegetativo. Para todos los tratamientos se utilizaron $2,5 \mathrm{~L}$ de agua de mar con una salinidad de 24 PSU, fotoperíodo 12:12 (L: O) y una intensidad lumínica de 8,3 $( \pm 3) \mu \mathrm{mol} \mathrm{m} \mathrm{m}^{-2} \mathrm{~s}^{-1}$. En el caso de la incorporación de fertilizantes, se determinó una combinación de fertilizantes comerciales como Anasac Fert Plant Floracion (AFP) y Best Garden Floración (BG), ambos en concentraciones de $40 \mathrm{ml} / \mathrm{L}$. El tratamiento con incorporación de provasoli considero adición de $20 \mathrm{ml} / \mathrm{L}$ de medio de cultivo. Las mediciones se realizaron cada 30 días aproximadamente durante un total de 151 días (inicio 10-05-12; medición 1: 08-06-12; medición 2: 06/07/12; medición 3: 03/08/12, medición 4: 07/09/12; medición final 07/10/12). Aquí se midieron las variables de biomasa húmeda (g) y la longitud de los talos $(\mathrm{cm})$. Se estimó el porcentaje de crecimiento diario (\% por día ${ }^{-1}$ ) con el protocolo de Hansen (1980): TDC $(\%)=100^{*} \ln \left(\mathrm{N}_{1} / \mathrm{N}_{0}\right) / \mathrm{t}$. Donde TDC $(\%)=$ Tasa de crecimiento diario en porcentaje $\left(\%\right.$ dia $\left.^{-1}\right) ; \mathrm{N}_{0}=$ Biomasa inicial; $\mathrm{N}_{1}=$ Biomasa final; intervalo de tiempo en días.

\section{Cultivo piloto en mar}

El sistema de cultivo piloto de A. plicata 


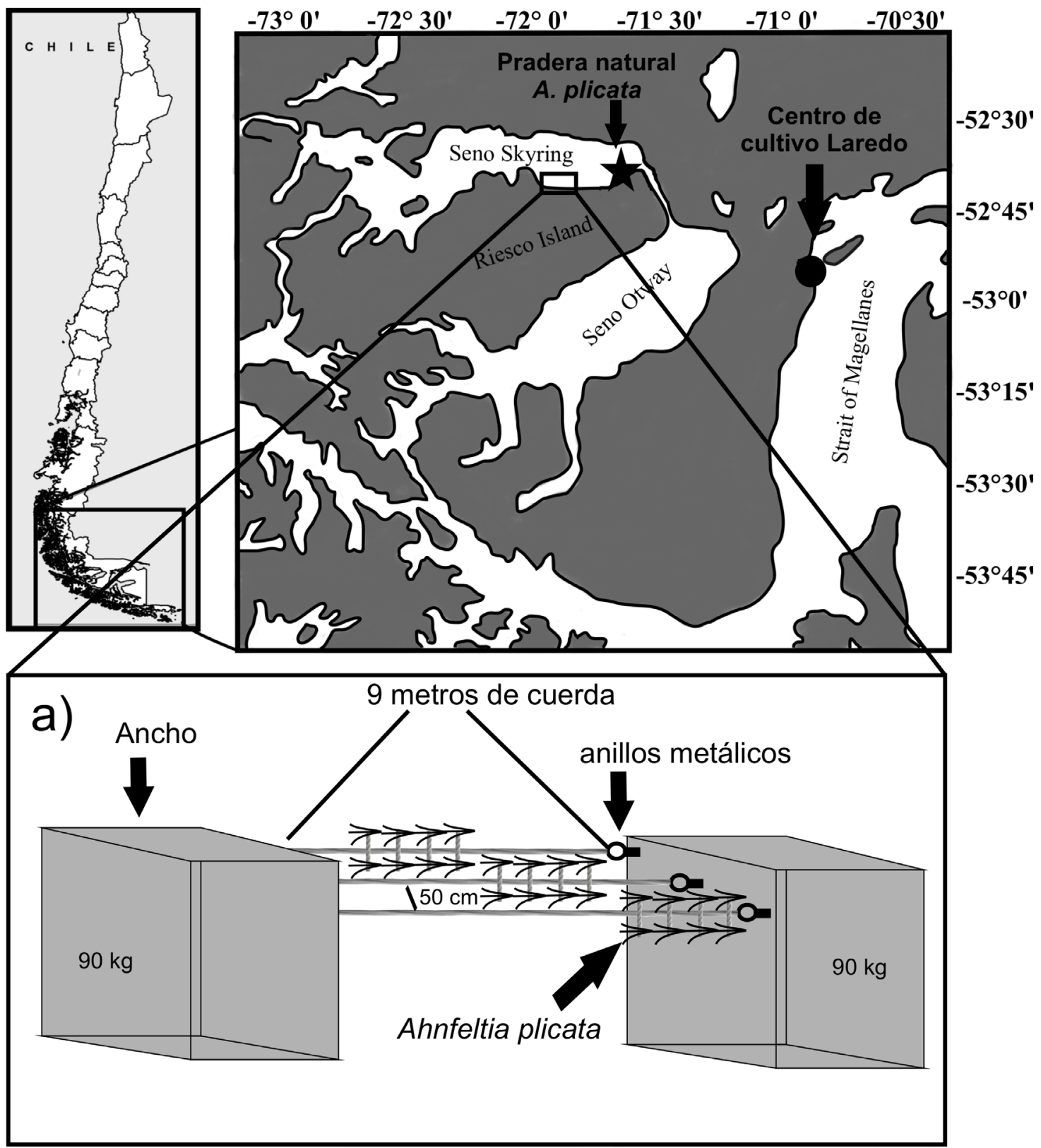

Fig. 1. Sitios de estudio de A. plicata en canales y fiordos de Magallanes, Chile. a) Esquema del sistema de cultivo Longline instalado para la evaluación de crecimiento en Seno Skyring (52³9'S; $\left.71^{\circ} 30^{\prime} \mathrm{W}\right)$.

fue realizado en seno Skyring en la costa de isla Riesco (52³9'S; 71³0’W) (Fig. 1a). Esta zona posee singularidades ecosistémicas diferentes a otros canales de Magallanes, por al menos dos razones: i) Su historia geológica da cuenta que fue un lago post-glacial y ii) presenta salinidades superficiales que fluctúan entre 17 a 18 psu.
La temperatura superficial de mar fluctúa entre $4,5^{\circ} \mathrm{C}$ en invierno y $10^{\circ} \mathrm{C}$ en verano (Kilian et al. 2007). Las variables de la luz cambian estacionalmente, por ejemplo, la radiación solar en invierno es de $46,6 \mathrm{Wm}^{-2}$, mientras que en verano es de 256,6 $\mathrm{Wm}^{-2}$ (Butorovic, 2012).

Para evaluar el crecimiento vegetativo se 
instaló un sistema de cultivo Long-line (Fig. 1a), donde se encordaron 72 talos infértiles de 1,3 g. La denominación infértil se categorizó cuando en los talos no se observaba macroscópicamente la presencia de estructuras reproductivas (nematecios). Las variables medidas fueron: biomasa húmeda (g) por talo, ocurrencia de nuevos tejidos (ramificaciones), presencia o ausencia de estructuras reproductivas y porcentaje de sobrevivencia. También se evaluó la tasa de crecimiento diario para cada mes, el protocolo utilizado fue el propuesto por Hansen (1980). Las mediciones se realizaron in situ cada 30 días desde mayo (2012) a enero (2013) (i.e., 9 meses). Las comparaciones temporales de crecimiento en biomasa por talo se evaluaron mediante un análisis no paramétrico de KruskalWallis, utilizando el programa estadístico STATISTICA 7.0.

\section{RESULTADOS}

\section{Cultivo en hatchery}

El experimento de cultivo vegetativo de A. plicata en hatchery mostró un bajo crecimiento vegetativo durante 151 días. Por ejemplo, el tratamiento con incorporación con Provasoli fue el que obtuvo mayor aumento en biomasa húmeda por talo, con un promedio inicial de 5,2 $\pm 0,15 \mathrm{~g}$ y tras 151 días alcanzo $6,1 \pm 0,1 \mathrm{~g}$ de biomasa húmeda. A su vez, para la variable longitud $(\mathrm{cm})$, el tratamiento con Provasoli también fue la que obtuvo mejores resultados, por ejemplo, el promedio de longitud inicial fue de $12,5 \pm 0,69 \mathrm{~cm}$ y tras 151 días alcanzo un promedio de $13,9 \pm 0,89 \mathrm{~cm}$. Por otro lado, si consideramos la tasa de crecimiento mensual en biomasa, los mayores valores se obtuvieron durante el segundo mes de medición con una tasa de 0,223\% dia ${ }^{-1}$ para el tratamiento de Provasoli (ver Tabla 1). En cambio, la tasa de crecimiento en longitud de talo, presentó mayores valores durante el primer intervalo de tiempo con el tratamiento de Fertilizantes comerciales con un $0,272 \%$ dia $^{-1}$ (ver Tabla 1 ). Cabe destacar, que a partir del tercer mes de cultivo en hatchery las tasas de crecimiento en todos los tratamientos comenzaron a disminuir incluso obteniendo valores negativos en tasas de crecimiento, tanto para biomasa húmeda y longitud de talos.

Finalmente, al evaluar el crecimiento diario considerando el total del periodo de tiempo del experimento (i.e., 151 días), se obtuvo que el tratamiento incorporación de Provasoli, evidencio la mejor tasa de crecimiento diario para ambas variables respuesta. Por ejemplo, la biomasa húmeda mostró una tasa crecimiento diario de $0,101 \%$, mientras que la menor tasa de crecimiento fue para el control con $0,031 \%$, a pesar de ello no se encontraron diferencias significativas entre los tratamientos (K-W: $\mathrm{H}=4,3 ; \mathrm{P}>0,05)$. En el caso de la longitud del talo, el tratamiento Provasoli evidencio la mayor tasa de crecimiento diario de 0,069\%, mientras que la menor tasa fue para el control con $0,019 \%$ (ver Fig. 2). Sin embargo, no se encontraron diferencias significativas entre los

Tabla 1. Tasa promedio de crecimiento diario (\% por día $\left.{ }^{-1}\right)$ entre cada intervalo de medición, para las variables biomasa húmeda (g) y longitud por talo $(\mathrm{cm})$ en los tratamientos control, incorporación de Provasoli y incorporación de Fertilizantes comerciales.

\begin{tabular}{lccccc}
\hline \multicolumn{5}{c}{ Intervalos de medición mensual } \\
\hline Biomasa & $10 / 05$ a 08/06 & $08 / 06$ a 06/07 & $06 / 07$ a 03/08 & 03/08 a 07/09 & $07 / 09$ a 07/10 \\
\hline Control & 0,164 & 0,102 & $-0,229$ & 0,118 & 0,017 \\
Provasoli & 0,144 & 0,223 & $-0,061$ & 0,079 & 0,111 \\
Fertilizante & 0,202 & 0,215 & $-0,217$ & 0,04 & $-0,005$ \\
\hline Longitud & & & & \\
\hline Control & 0,025 & 0,002 & 0,076 & 0,001 & $-0,003$ \\
Provasoli & 0,177 & 0,021 & 0,052 & 0,018 & 0,077 \\
Fertilizante & 0,272 & $-0,024$ & 0,094 & 0,002 & 0,007 \\
\hline
\end{tabular}


tratamientos (K-W: $\mathrm{H}=2,4 ; \mathrm{P}>0,05)$.

\section{Cultivo piloto en mar}

El crecimiento vegetativo de $A$. plicata se inicio con $1,3 \mathrm{~g} /$ talo (mayo 2012) y finalizó con una biomasa promedio de 2,2 g/talo (enero 2013). Por lo tanto, el crecimiento total por talo en biomasa fue de 0,9 g en 9 meses (ver Fig. 3). Las diferencias significativas del incremento en biomasa se observaron sólo en los dos últimos meses (diciembre y enero) en comparación con la biomasa inicial (K-W: $\mathrm{H}=73,4 ; \mathrm{P}<0,05)$. La mayor tasa de crecimiento promedio mensual se observó en diciembre (verano austral) $0,52 \%$ y la menor tasa en agosto (invierno austral) con $-0,24 \%$, durante este periodo se detecto perdida de biomasa en algunos individuos.

Otro parámetro relacionado con el crecimiento de A. plicata, fue la detección de nuevos tejidos. Es decir, ramificaciones de crecimiento que comenzaron a observarse desde septiembre en un $45 \%$ de los talos, incrementándose durante enero con $82 \%$ de los talos (Fig. 4a). En cuanto a los parámetros reproductivos, se observó que al octavo mes de cultivo (diciembre) un $56 \%$ de los talos

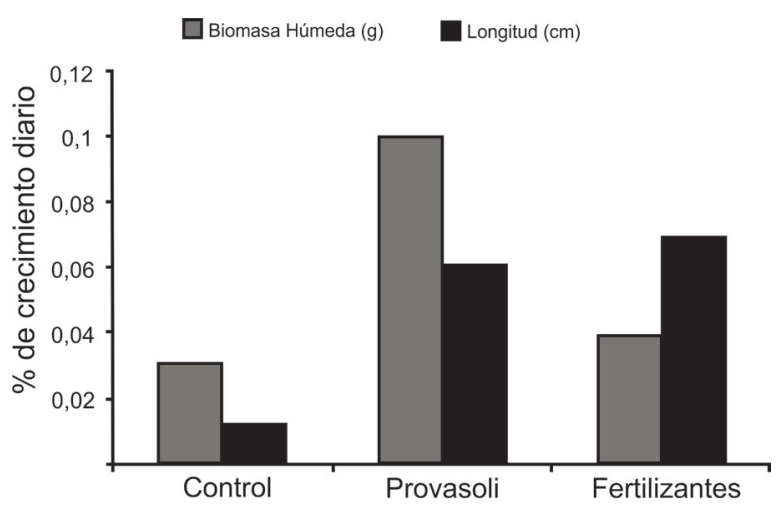

Fig. 2. Porcentaje de crecimiento diario de A. plicata, durante 151 días de cultivo vegetativo en tres tipos de tratamiento experimental en hatchery.

presentaron estructuras reproductivas (nematecios), estos individuos fueron clasificados como fértiles (Fig. 4b). La sobrevivencia de los talos monitoreados durante los 9 meses fue de un 100\%. Cabe destacar, que entre septiembre y octubre (primavera austral) se detectó un crecimiento explosivo de epifitos del género Ectocapus sobre los talos y el sistema de cultivo, debiendo realizar un manejo mediante la limpieza de epifitos en los individuos de A. plicata como también en el sistema de cultivo.

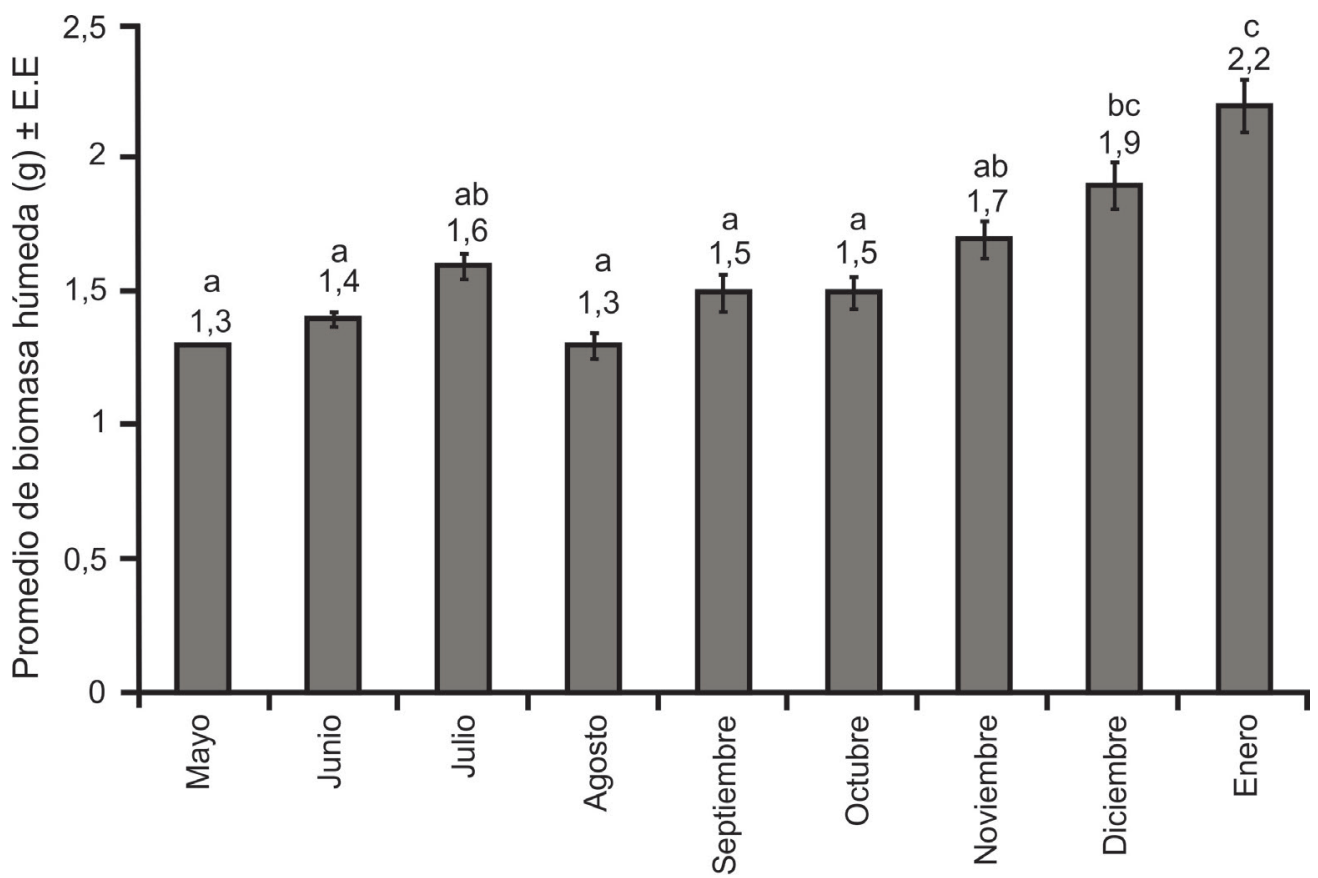

Fig. 3. Promedio mensual de biomasa húmeda $(\mathrm{g}) \pm \mathrm{S}$.E de talos de A. plicata instalados en sistema Long-line en Seno Skyring (52 $40^{\prime}$ S y $\left.71^{\circ} 54^{\prime} \mathrm{O}\right)$. Las letras distintas indican diferencias estadísticamente significativas $(p<0,05)$. 


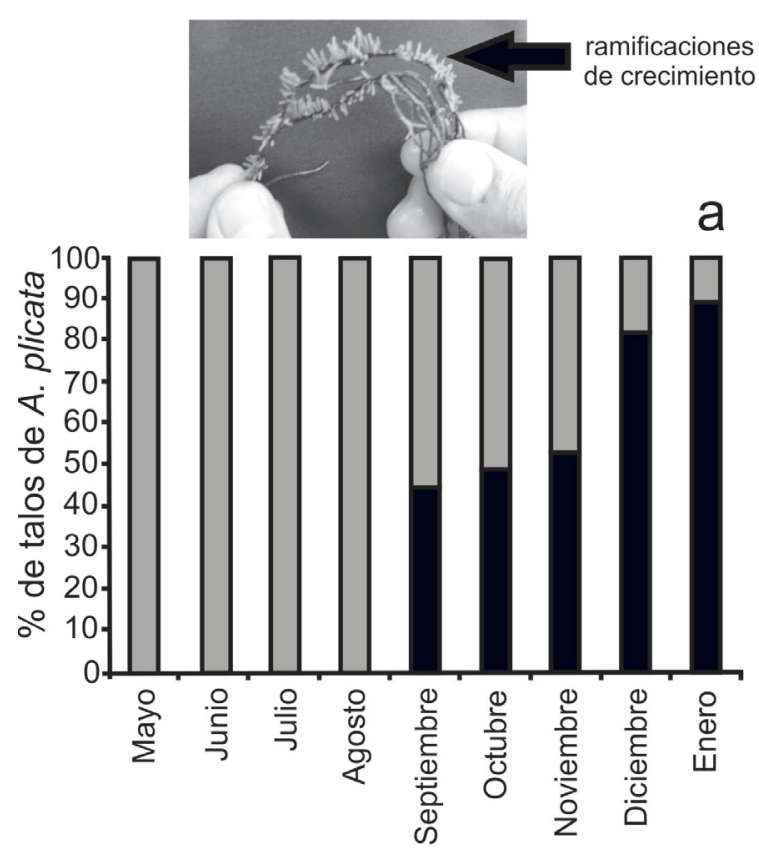

sin ramificaciones

Con ramificaciones
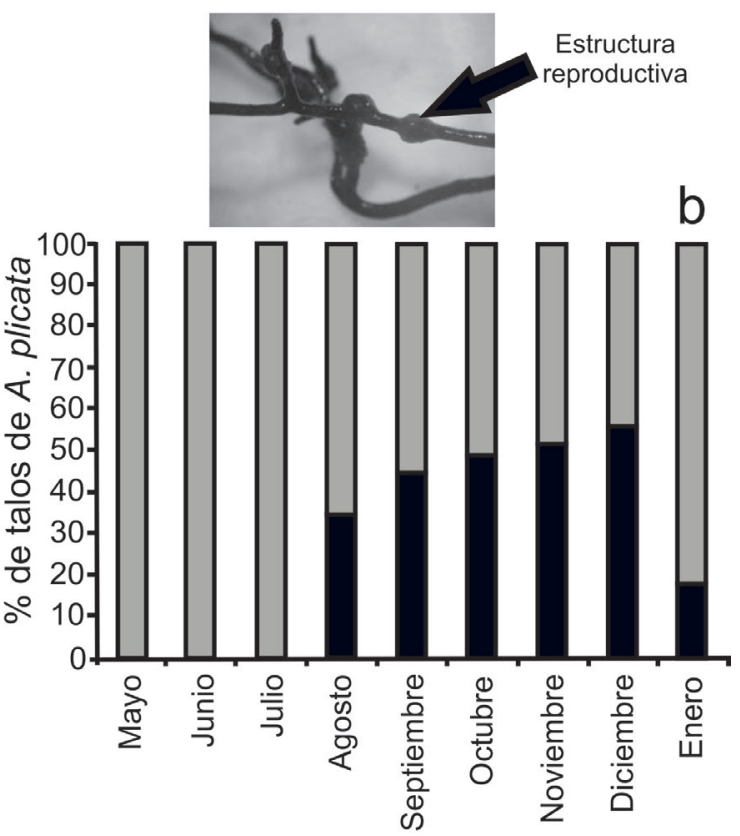

Infertil

Fertil

Fig. 4. Variación temporal de crecimiento y estado reproductivo para 72 talos de A. plicata en un sistema de cultivo vegetativo en seno Skyring. a) Porcentaje mensual de ramificaciones nuevas. b) Porcentaje mensual de individuos fértiles e infértiles.

\section{DISCUSIÓN}

La agarofita $A$. plicata tiene un gran potencial como especie comercial para la industria de los ficocoloides en Chile, principalmente debido a que presenta una agarosa neutra libre de grupos sulfatos (Matsuhiro et al. 2014). A su vez, existe un gran desafío de crear metodologías de cultivo por al menos dos razones: i) incentivar su producción de agarosa, y ii) no afectar la sustentabilidad de las praderas naturales en la región de Magallanes. No obstante, experiencias de cultivo en laboratorio han mostrado que presenta un lento crecimiento (e.g., Chen, 1977). Nuestros datos de cultivo vegetativo a escala de harchery y en mar, también concuerdan con este lento crecimiento. Por ejemplo, en hatchery la mejor tasa de crecimiento fue de 0,101\% diario en 151 días de cultivo para el tratamiento en hatchery. Incluso si consideramos la tasa de crecimiento diario durante un periodo mensual, los mejores resultados fueron obtenidos para el tratamiento de incorporación de fertilizantes comerciales con $0,215 \%$ diario. A su vez, nuestros datos de cultivo en mar muestran que la máxima tasa de crecimiento diario mensual de A. plicata fue de 0,52\% durante el mes de diciembre. Estos valores de producción de biomasa húmeda y longitud por talo evidencian que A. plicata tiene un lento crecimiento bajo condiciones de cultivo vegetativo, comparado con otra agarofita como G. chilensis que puede alcanzar una tasa de crecimiento en biomasa de un $7 \%$ diario en un mes de monitoreo (ver Troell et al. 1997). Este crecimiento lento de A. plicata, probablemente se debe a la ontogenia de esta macroalga, ya que otras experiencia con estimuladores de crecimiento hormonales presentan datos similares (Mansilla et al. 2013). A pesar de ello, consideramos que este sistema de cultivo mantiene y provee buenas condiciones para el crecimiento de los talos, por al menos cuatro razones: i) no presenta mortalidad; ii) los talos presentaron formación de estructuras reproductivas; iii) se observó la aparición de nuevas ramificaciones y iv) en el cultivo en mar durante los meses estivales se obtuvieron tasas de crecimiento más altas, que las obtenidas en 151 días de cultivo en 
hatchery con condiciones controladas. Finalmente, esta es la primera experiencia de cultivo piloto en mar y hatchery para $A$. plicata en canales $y$ fiordos subantárticos, mostrando indicadores de lento crecimiento. Por esta razón, una propuesta de cultivo productivo de tipo vegetativo a escala de masiva es aún una tarea compleja para el cultivo de macroalgas en la región de Magallanes. No obstante, es necesario estudiar nuevas estrategias que permitan el uso sustentable de este nuevo $e$ importante recurso que diversifica la utilización de especie de importancia comercial para el sur austral de Chile.

\section{AGRADECIMIENTOS}

Al proyecto FONDEF D08I1163 otorgado por la Comisión de Ciencia y Tecnología de Chile. Al equipo de trabajo Johanna Marambio, Alejandra Beattie, Jorge Balic, Constanza Jiménez, Juan Rodríguez, Fabio Méndez, Paula Ocaranza, Javier Rendoll y José Solís. También agradecemos por su apoyo al Instituto de Ecología y Biodiversidad (IEB).

\section{LITERATURA CITADA}

Astorga-España, M. S., \& Mansilla, A. (2013). Sub-Antarctic macroalgae: opportunities for gastronomic tourism and local fisheries in the Region of Magallanes and Chilean Antarctic Territory. Journal Applied Phycology DOI 10.1007/s10811-013-0141-1.

Bulboa, C., \& Macchiavello, J. (2006). Cultivation of cystocarpic, tetrasporic and vegetative fronds of Chondracanthus chamissoi (Rhodophyta, Gigartinales) on ropes at two localities in northern Chile. Investigaciones Marinas, 34, 109-112.

Buschmann, A. H., Correa, J. A., Westermeier, R., Hernández-González, M. C., \& Norambuena, R. (2001). Red algal farming in Chile: a review. Aquaculture, 194, 203220.

Butorovic, N. (2013). Resumen meteorológico año 2012, Estación "Jorge C. Schythe". Anales del Instituto de la Patagonia 41: 153-162.

Camus, P. A., \& Barahona, R. M. (2002). Intertidal insects from Concepción, Chile: perspectives for ecological research. Revista Chilena de
Historia Natural, 75, 793-803.

Chen L. C. M. (1977). The sporophyte of Ahnfeltia plicata (Huds.) Fries (Rhodophyceae, Gigartinales) in culture. Phycologia, 16,163168.

Hansen, J. E. (1980). Physiological considerations in the mariculture of red algae. In: Abbott, I. A., Foster, M.S., \& Eklund, L.F. (eds), Pacific seaweed Aquaculture (pp.80-91. La Jolla: Publ. California Sea Grant Coll. Program.

Hori, M., Noda, T., \& Nakao, S. (2006). Effects of avian grazing on the algal community and small invertebrates in the rocky intertidal zone. Ecological Research, 21, 768-775.

Kilian, R., Baeza, O., Steinke, T., Arevalo, M., Ríos, C., \& Schneider, C. (2007). Late Pleistocene to Holocene marine transgression and thermohaline control on sediment transport in the western Magellanes fjord system of Chile (53S). Quaternary International, 161, 90-107.

Maggs, C. A., \& Pueschel, C. M. (1989). Morphology and development of Ahnfeltia plicata (Rhodophyta): proposal of Ahnfeltiales ord. nov. Journal Phycology, 25, 333-351.

Mansilla, A., Ávila, M., \& Yokoya, N. S. (2012). Current knowledge on biotechnological interesting seaweeds from the Magellan Region, Chile. Brazilian Journal of Pharmacognosy, 22, 760-767.

Mansilla, A., Rodríguez, J. P., Souza, J., Rosenfeld, S., Ojeda, J., \& Yokoya, N. S. (2013). Growth responses to temperature, salinity and nutrient variations, and biomass variation and phenology of Ahnfeltia plicata (Rhodophyta, Ahnfeltiales), a comercial interesting agarophyte from the Magellan Region, Chile. Journal Applied Phycology DOI 10.1007/s10811-013-0150-0.

Matsuhiro, B., Barahona, T., Encinas, M. V., Mansilla, A., \& Ortiz J. A. (2014). Sulfation of agarose from subantarctic Ahnfeltia plicata (Ahnfeltiales, Rhodophyta): studies of its antioxidant and anticoagulant properties in vitro and its copolymerization with acrylamide Journal Applied Phycology DOI 10.1007/s10811-014-0297-3. 
Morya, V. K., Kim, J., \& Kim, E. (2012). Algal Fucoidan: strutural and size-dependet bioactivities and their perspectives. Applied Microbiology and Biothenology 93: 71-82.

Troell, M., Halling, C., Nilsson, A., Buschmann, A. H., Kautsky, N., \& Kautsky, L. (1997). Integrated marine cultivation of Gracilaria chilensis (Gracilariales, Rhodophyta) and salmon cages for reduced environmental impact and increased economic output. Aquaculture, 156: 45-61.

Villanueva, F., Ávila. M., Mansilla, A., Abades,
S., \& Cáceres, J. 2013. Efecto de auxinas y citoquininas en el cultivo de tejido de Ahnfeltia plicata (Hudson) Fries, 1836 (Ahnfeltiales, Rhodophyta) de la región de Magallanes. Anales del Instituto de la Patagonia, 41, 99-111.

Westermeier, R., Rivera, P. J., \& Gómez, I. (1991). Cultivation of Gracilaria chilensis Bird, McLachlan and Oliveira, in the intertidal and subtidal zones of Cariquilda estuary, Maullín, Chile. Revista Chilena de Historia Natural, 64, 307-321. 
